\title{
Injury to Inferior Vena Cava
}

National Cancer Institute

\section{Source}

National Cancer Institute. Injury to Inferior Vena Cava. NCI Thesaurus. Code C78363.

Damage to the inferior vena cava from traumatic or pathologic processes. 